\title{
Threshold of transverse mode coupling instability with arbitrary space charge
}

\author{
V. Balbekov* \\ Fermi National Accelerator Laboratory, P.O. Box 500, Batavia, Illinois 60510, USA
}

(Received 3 August 2017; published 30 November 2017)

\begin{abstract}
The threshold of the transverse mode coupling instability is calculated in framework of the square well model at arbitrary value of space charge tune shift. A new method of calculation is developed beyond the traditional expansion technique. The square, resistive, and exponential wakes are investigated. It is shown that the instability threshold goes up indefinitely when the tune shift increases. A comparison with conventional case of the parabolic potential well is performed.
\end{abstract}

DOI: 10.1103/PhysRevAccelBeams.20.114401

\section{INTRODUCTION}

The transverse mode coupling instability (TMCI) of a bunch with space charge (SC) was considered in papers [1-6]. The authors have represented similar results concerning the SC impact on the TMCI at a moderate ratio of the $\mathrm{SC}$ tune shift to the synchrotron tune $\left(\Delta Q / Q_{s}\right)$. It follows from these papers that the threshold of the instability caused by a negative wake increases when the ratio goes up from zero to several tens, at least.

However, more confusing pictures appear at larger (but realistic) values of this ratio like a hundred or over it. It has been suggested in Ref. [2] that the threshold growth ceases above this border becoming to 0 at $\Delta Q / Q_{s} \rightarrow \infty$. By contrast, it was asserted in Ref. [3] that negative wake cannot excite the TMCI in this limiting case.

It should be noted in this regard that approximate methods of solution were applied in all quoted articles. Typically, expansion of the coherent bunch displacement in terms of some set of basic vectors, with subsequent truncation of the series, has been used at the modest tune shift. However, as it has been shown later in Ref. [6], the number of the equations should be approximately proportional to $\Delta Q / Q_{s}$ to provide appropriate convergence of results with negative wake. Therefore, a smooth asymptotic transition is really impossible in the framework of this method. In contrast, the convergence is very well with positive wake allowing to reach the result by using the three-mode approximation $[5,6]$.

The method which is developed in the present article does not use the expansion at all, and allows us to get the TMCI thresholds as a smooth function of arbitrary SC tune

\footnotetext{
*balbekov@fnal.gov
}

Published by the American Physical Society under the terms of the Creative Commons Attribution 4.0 International license. Further distribution of this work must maintain attribution to the author(s) and the published article's title, journal citation, and DOI. shift. In the beginning of the paper, it is applied to the square potential well model. Such a model has been used earlier together with the expansion technique to investigate the TMCI at modest space charge [1,2]. More wide results are represented in this article. In particular, it is shown that the TMCI threshold with negative wake is about proportional to $\Delta Q / Q_{s}$. It is shown as well that the conclusion still stands with resistive and exponential wakes.

\section{PHYSICAL MODEL}

\section{A. General relations}

The chromaticity will not be considered in this paper because it is a factor of small importance for the TMCI [5]. Then the transverse coherent displacement of a bunch in the rest frame can be represented as the real part of the function

$$
X(\theta, u, t)=Y(\theta, u) \exp \left[-i Q_{\beta}\left(\theta+\Omega_{0} t\right)-i \Omega_{0} \nu t\right]
$$

where $\theta$ and $u$ are the coordinate and the momentum of a particle in the longitudinal phase space, $t$ is time, $Q_{\beta}$ and $\Omega_{0}$ are the central betatron tune and the revolution frequency, respectively, $\nu$ is an additional tune shift due to the wake field. The wake function will be characterized in the paper by the function $q(\theta)$. Then the function $Y$ satisfies the equation

$$
\begin{aligned}
\nu Y & +i Q_{s} \frac{\partial Y}{\partial \phi}+\Delta Q(Y-\bar{Y}) \\
& =2 \int_{\theta}^{\infty} q\left(\theta^{\prime}-\theta\right) \bar{Y}\left(\theta^{\prime}\right) \rho\left(\theta^{\prime}\right) d \theta^{\prime}
\end{aligned}
$$

where $\phi$ and $Q_{s}$ are the phase and the tune of the synchrotron oscillations, and $\Delta Q$ is the space charge produced betatron tune shift [3]. The variable $\bar{Y}$ is defined by the relations 


$$
\begin{aligned}
\rho(\theta) \bar{Y}(\theta) & =\int_{-\infty}^{\infty} \Phi(\theta, u) Y(\theta, u) d u, \\
\rho(\theta) & =\int_{-\infty}^{\infty} \Phi(\theta, u) d u
\end{aligned}
$$

with $\Phi$ as the normalized distribution function of the bunch.

The function $q(\theta)$ is proportional to the transverse wake potential $W_{1}(z)$ where $z=R \theta$ and $R$ is the machine radius:

$$
q(\theta)=\frac{r_{0} N_{b} R W_{1}(-R \theta)}{8 \pi \beta \gamma Q_{\beta}}
$$

with $r_{0}=e^{2} / m c^{2}$ as the particle electromagnetic radius, $\beta$ and $\gamma$ as its normalized velocity and energy, and $N_{b}$ as the bunch population [5]. It is convenient to split the function into 2 multipliers characterizing the wake normalized amplitude and its shape: $q(\theta)=q_{0} w(\theta)$ where

$$
\begin{gathered}
q_{0}=2 \int_{-\infty}^{\infty} \rho(\theta) d \theta \int_{\theta}^{\infty} q\left(\theta^{\prime}-\theta\right) \rho\left(\theta^{\prime}\right) d \theta^{\prime}, \\
1=2 \int_{-\infty}^{\infty} \rho(\theta) d \theta \int_{\theta}^{\infty} w\left(\theta^{\prime}-\theta\right) \rho\left(\theta^{\prime}\right) d \theta^{\prime} .
\end{gathered}
$$

With this definition, the tune of the lowest (rigid) bunch mode is

$$
\nu_{\text {rigid }}=q_{0} \quad \text { at } Y=\bar{Y}=1 \text {. }
$$

Of course, this expression can be valid at full only at $q_{0} \ll Q_{s}$ when coupling of the modes is negligible. However, it does not matter in this consideration because the value is used merely for the normalization.

Separating the even and the odd parts of the function $Y=Y^{+}(\phi)+Y^{-}(\phi)$, one can get the equation

$$
\begin{aligned}
\hat{\nu} Y^{+} & +Q_{s}^{2} \frac{\partial}{\partial \phi}\left(\frac{\partial Y^{+}}{\hat{\nu} \partial \phi}\right) \\
& =\Delta Q \bar{Y}+2 \int_{\theta}^{\infty} q\left(\theta^{\prime}-\theta\right) \bar{Y}\left(\theta^{\prime}\right) \rho\left(\theta^{\prime}\right) d \theta^{\prime}
\end{aligned}
$$

where $\hat{\nu}(\theta)=\nu+\Delta Q(\theta)$.

\section{B. Square potential well}

In practice, it is more convenient to use a variable $\vartheta \propto \theta$ as the longitudinal coordinate adjusted to the bunch length. For the square well model used in the paper, the best choice is the well and the bunch location in the interval $0<\vartheta<\pi$. Then

$$
\vartheta=|\phi|, \quad \phi=\vartheta \frac{u}{|u|}, \quad \rho(\vartheta)=\frac{1}{\pi} \text { at } 0<\vartheta<\pi .
$$

Note that Eq. (7) remains in force with the new variable if the normalization conditions Eq. (5) are adjusted as well to save the validity of Eq. (6).

Any monotonous function of $|u|$ can be used as the synchrotron amplitude in the case. The synchrotron tune $Q_{S}$ is the most natural and convenient choice. Therefore, taking into account that only $Y^{+}$makes the contribution into Eq. (3a), and that it is an even function of $u$, one can rewrite this expression in the form

$$
\bar{Y}(\vartheta)=\int_{0}^{\infty} F\left(Q_{s}\right) Y^{+}\left(\vartheta, Q_{s}\right) d Q_{s}
$$

with the normalization condition

$$
\int_{0}^{\infty} F\left(Q_{s}\right) d Q_{s}=1
$$

Because $Y^{+}\left(\phi, Q_{s}\right)$ is an even and periodic function of $\phi$, it is sufficient to consider the interval $0<\phi<\pi$ where $\phi=\vartheta$. Taking into account as well that $\Delta Q=$ const in the square potential well, one can represent Eq. (7) in the form

$Q_{s}^{2} \frac{\partial^{2} Y^{+}}{\partial \vartheta^{2}}+\hat{\nu}^{2} Y^{+}=\hat{\nu} \Delta Q \bar{Y}+\frac{2 \hat{\nu}}{\pi} \int_{\vartheta}^{\pi} q\left(\vartheta^{\prime}-\vartheta\right) \bar{Y}\left(\vartheta^{\prime}\right) d \vartheta^{\prime}$.

The boundary conditions of the equation are

$$
\frac{\partial Y^{+}}{\partial \theta}\left(0, Q_{s}\right)=\frac{\partial Y^{+}}{\partial \theta}\left(\pi, Q_{s}\right)=0
$$

whose relation also follows from the periodicity and the parity of the function $Y^{+}$.

\section{HOLLOW BUNCH WITH A SQUARE WAKE}

A hollow bunch will be considered below. Its distribution function is

$$
F\left(Q_{s}\right)=\delta\left(Q_{s}-Q_{s 0}\right)
$$

According to Eq. (9), $\bar{Y}(\vartheta)=Y^{+}\left(\vartheta, Q_{s 0}\right)$ in this case, so that Eq. (11) and its boundary conditions Eq. (12) leads to the expression

$$
\begin{gathered}
Q_{s 0}^{2} \bar{Y}^{\prime \prime}(\vartheta)+\hat{\nu}(\hat{\nu}-\Delta Q) \bar{Y}(\vartheta)=\frac{2 \hat{\nu}}{\pi} \int_{\vartheta}^{\pi} q\left(\vartheta^{\prime}-\vartheta\right) \bar{Y}\left(\vartheta^{\prime}\right) d \vartheta^{\prime} \\
\bar{Y}^{\prime}(0)=\bar{Y}^{\prime}(\pi)=0 .
\end{gathered}
$$

As the first step, we consider the simplest case of constant wake: $q=q_{0}, w=1$ inside the bunch. It results in the equation 


$$
\bar{Y}^{\prime \prime}(\vartheta)+\frac{\hat{\nu}(\hat{\nu}-\Delta Q)}{Q_{s 0}^{2}} \bar{Y}(\vartheta)=\frac{2 \hat{\nu} q_{0}}{\pi Q_{s 0}^{2}} \int_{\vartheta}^{\pi} \bar{Y}\left(\vartheta^{\prime}\right) d \vartheta^{\prime}
$$

Only the case $q_{0} \leq 0$ will be investigated below because the positive wake is an occasional and not questionable occurrence $[5,6]$.

\section{A. Solution by an expansion}

This subsection pursues two goals. The first of them is a clarification of some properties of the solution for a further usage, and second one is a comparison with the more conventional case of a parabolic potential well where the expansion technique is the prevailing method.

A general solution of Eq. (15) with boundary conditions given by Eq. (14b) can be represented as the series

$$
\bar{Y}(\vartheta)=\sum_{n=0}^{\infty} Y_{n} \cos n \vartheta
$$

with unknown coefficients $Y_{n}$. Then the equation rearranges to the form

$$
\begin{gathered}
\sum_{n=0}^{\infty}\left[\hat{\nu}(\hat{\nu}-\Delta Q)-n^{2} Q_{s 0}^{2}\right] Y_{n} \cos (n \vartheta) \\
=\frac{2 q_{0} \hat{\nu}}{\pi} \sum_{n=0}^{\infty} Y_{n} \int_{\theta}^{\pi} \cos \left(n \vartheta^{\prime}\right) d \vartheta^{\prime} .
\end{gathered}
$$

Multiplying this expression by $\cos (N \vartheta)$ and integrating over $\theta$, one can obtain the series of equations for the coefficients $Y_{n}$ :

$\left[\hat{\nu}(\hat{\nu}-\Delta Q)-N^{2} Q_{s 0}^{2}\right] Y_{N}=q_{0} \hat{\nu}\left(2-\delta_{N, 0}\right) \sum_{n=0}^{\infty} R_{N, n} Y_{n}$

where $R_{n, n}=\delta_{n, 0}$, and other elements of the $R$-matrix are

$$
\begin{aligned}
R_{N, n} & =\frac{2}{\pi^{2}} \int_{0}^{\pi} \cos (N \vartheta) d \vartheta \int_{\theta}^{\pi} \cos \left(n \vartheta^{\prime}\right) d \vartheta^{\prime} \\
& =\frac{2\left[1-(-1)^{N-n}\right]}{\pi^{2}\left(N^{2}-n^{2}\right)} .
\end{aligned}
$$

The lower order terms of $R$-matrix are shown in Table I.

The infinite series given by Eq. (18) can be truncated by using of the assumption $Y_{n}=0$ at $n>N_{\max }$. It results in the finite set of the equations

$$
\begin{gathered}
\sum_{n=0}^{N_{\max }} T_{N, n} \bar{Y}_{n}=0, \\
T_{N, n}=q_{0} \hat{\nu}\left(\delta_{N, 0}-2\right) R_{N, n}+\left[\hat{\nu}(\hat{\nu}-\Delta Q)-N^{2} Q_{s 0}^{2}\right] \delta_{N, n} .
\end{gathered}
$$

TABLE I. The lower-order tems of the matrix $R_{N, n}\left(A=4 / \pi^{2}\right)$.

\begin{tabular}{ccccccc}
\hline \hline$-N \rightarrow$ & 0 & 1 & 2 & 3 & 4 & 5 \\
\hline$n=0$ & 1 & $A$ & 0 & $A / 9$ & 0 & $A / 25$ \\
$n=1$ & $-A$ & 0 & $A / 3$ & 0 & $A / 15$ & 0 \\
$n=2$ & 0 & $-A / 3$ & 0 & $A / 5$ & 0 & $A / 21$ \\
$n=3$ & $-A / 9$ & 0 & $-A / 5$ & 0 & $A / 7$ & 0 \\
$n=4$ & 0 & $-A / 15$ & 0 & $-A / 7$ & 0 & $A / 9$ \\
$n=5$ & $-A / 25$ & 0 & $-A / 21$ & 0 & $-A / 9$ & 0 \\
\hline \hline
\end{tabular}

A minimal set comes with $N_{\max }=0$ and includes only the lowest (rigid) head-tail mode $Y_{0}=1$. Equation (20) gives in this case $T_{0,0}=0$ that is $\hat{\nu}-\Delta Q=\nu=q_{0}$ as it is required by Eq. (6).

The general resolvability condition of the series is $\operatorname{det} T=0$ which is referred in the case to the algebraic equation of power $P=2\left(N_{\max }+1\right)$. It has $P$ roots which are the real numbers at $q_{0}=0[1,2]$ :

$$
\hat{\nu}_{n, \pm}=\frac{\Delta Q}{2} \pm \sqrt{\frac{\Delta Q^{2}}{4}+n^{2} Q_{s 0}^{2}}
$$

where $n=0,1, \ldots, N_{\max }$. Therefore, the following steps can be used to resolve the problem and to find the TMCI threshold at arbitrary $\Delta Q$ and $q_{0}: 1$. To select some values $N_{\max }$ and $\Delta Q / Q_{s 0}$. 2. To choose some trial value of $q_{0} / Q_{s 0} .3$. To find the matrix $T\left(\hat{\nu} / Q_{s 0}\right)$ and to calculate its determinant with given parameters and variable $\hat{\nu} / Q_{s 0}$. 4. To find number of real roots of the equation by counting how many times the determinant changes sign at increasing $\hat{\nu}$. 5. To repeat the procedure with higher value of $\left|q_{0} / Q_{s 0}\right|$ until the number of the real roots decreases. It will mean that a pair of complex roots appears in this point, and the reached value of $q_{0}$ is just the TMCI threshold with given $\mathrm{SC}$ tune shift at given approximation. 6 . To check the convergence of the results by comparison of the thresholds obtained with different $N_{\max }$.

Some results of the calculation are presented in Fig. 1 where the TMCI threshold of a negative wake is plotted against the tune shift at different $N_{\max }$. The black dropdown curve belongs to all the approximations. It is seen that any higher approximation follows the course which the lower ones have charted, and provides its continuation to higher $\Delta Q$. In contrast with it, the coming back lines of different color do not repeat each other so they cannot be treated as the credible results. It allows to conclude that, with negative wake, threshold value of $\left|q_{0}\right|$ is an increasing function of the SC tune shift in the considered range of $\Delta Q / Q_{0}$, and that rather large number of $N_{\max }$ is needed to reach the correct result with higher shift. Note that a coalescence of a pair of real roots in the $\left(q_{0}-\hat{\nu}\right)$ plane prefaces appearance of the complex roots The function $\hat{\nu}\left(q_{0}\right)$ satisfies the condition 


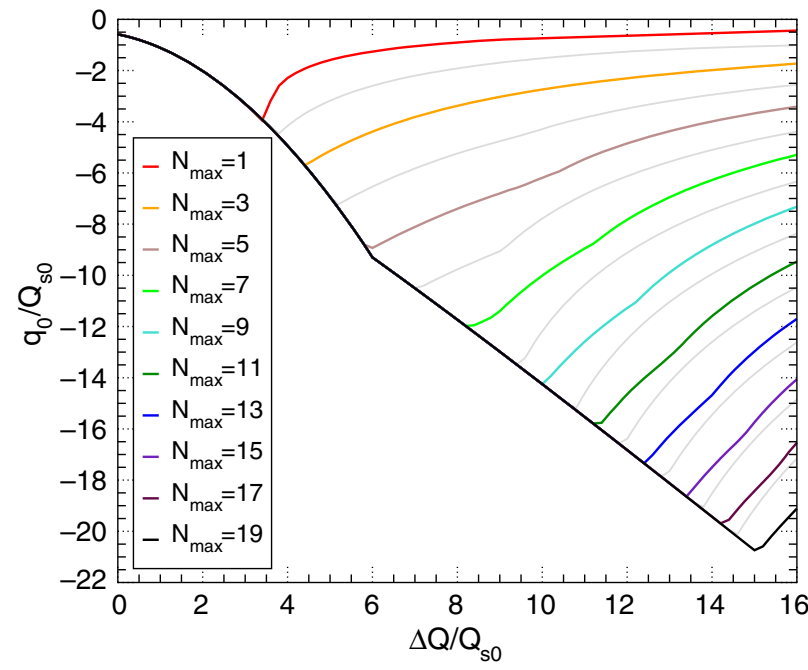

FIG. 1. The TMCI threshold of the hollow bunch in the square potential well against the SC tune shift. Different curves are obtained with different $N_{\max }=1, \ldots 19$. Each of them has a restricted region of applicability which expands with $N_{\max }$ growing. The drop-down parts of the curves merge forming the sole black line. The rising lines do not confirm each other marking ends of the applicability regions.

$$
\frac{d \hat{\nu}}{d q_{0}} \rightarrow \infty
$$

in the coalescence point. The condition is valid independently on $N_{\max }$ so it can be used to identify the instability threshold with any truncation of the expansion, or without the expansion at all.

Additional information is provided by Fig. 2 where complete observed bunch spectrum is plotted against $\Delta Q / Q_{s 0}$ at $N_{\max }=8$. The corresponding threshold value of $q_{0} / Q_{s 0}$ is plotted in the figure as well by the bold black line. As it should be in the threshold, there is a pair of coalesced spectral lines in any part of the plot. At $\Delta Q / Q_{s 0}<6$, they are presented as green lines which are identified as the multipoles $m=0$ and $m=-1$. The green lines diverge after $\Delta Q / Q_{s 0}>6$, and the instability transforms to a coalescence of the modes $m=-2$ and $m=-3$ (the blue lines). Finally, coalescence of the modes $m=1$ and $m=2$ appears at $\Delta Q / Q_{q}>9.5$ which case is presented by red lines. However, in contrast with previous pairs, this coalescence is not confirmed by calculations with higher $N_{\max }$. It allows to conclude that this coalescence is a nonphysical effect appearing out the region of applicability of the used approximation. The statement is confirmed by Fig. 3 where the tunes of the essential modes are plotted against SC tune shift at different $N_{\max }$. It is seen that the "responsibility" of the modes $m=-2$ and $m=-3$ for the instability extends to higher values of the tune shift (blue symbols).

It is important to emphasize a similarity of these results to those obtained with the model of parabolic potential

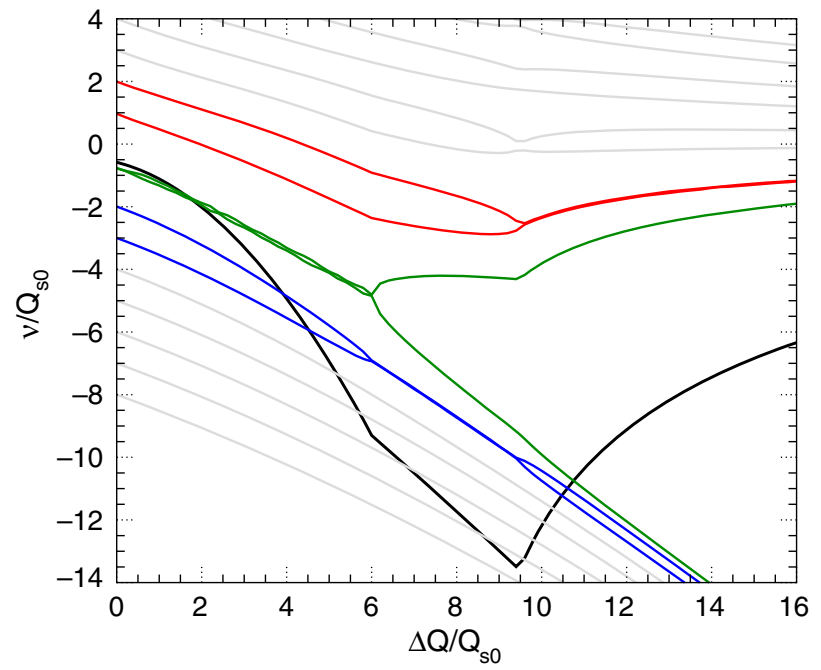

FIG. 2. Threshold bunch spectrum at $N_{\max }=8$. The solid black line is the TMCI threshold in this approximation. Other lines present the bunch eigentunes just before the instability appears. Coalescence of different multipoles is responsible for the instability at different $\Delta Q / Q_{s 0}$. The essential lines are emphasized by colors.

well [6]. Besides the general resemblance, there is a proximity of the numerical results. For example, applicability region of the approximation $N_{\max }=12$ is $\Delta Q / Q_{s 0}<12$ in both cases, and the calculated TMCI threshold is $q_{0} / Q_{s 0}=-16.7$ according to Fig. 1 and -18 according to [6]. It occurs in spite of the fact that the parabolic bunch has much richer spectrum than the square one, due to the higher radial modes which are absent in the later case. However, it has been shown in Ref. [6] that only lowest radial modes are capable to coalesce producing the TMCI. The square well model represents this part of the

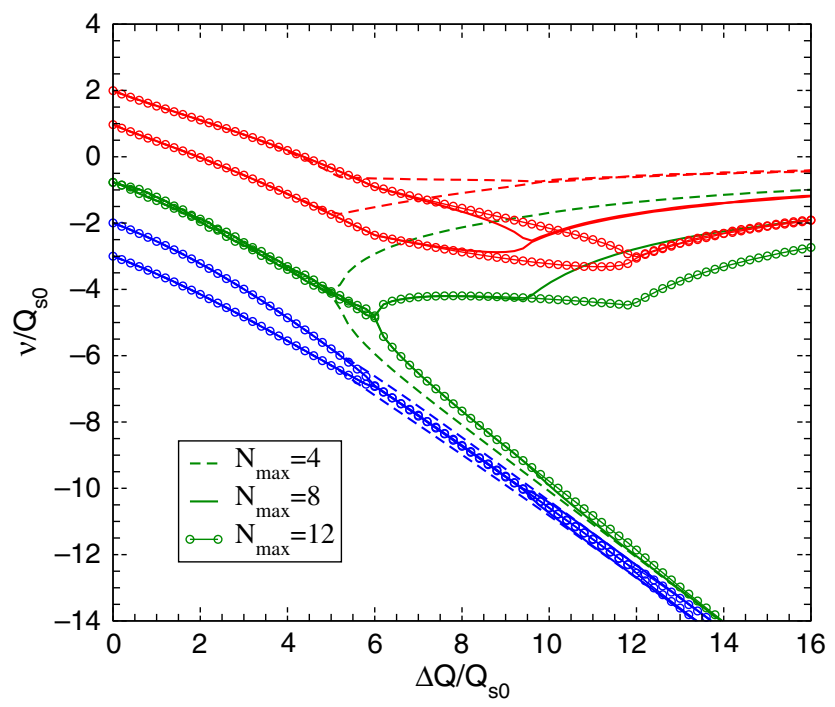

FIG. 3. The threshold bunch spectrum obtained with different $N_{\text {max }}$. Only essential (capable to coalesce) spectral lines are shown. 
spectrum rather correctly to calculate the valid TMCI threshold.

\section{B. Solution without expansion}

Another method of solution of Eq. (15) which is considered in this subsection is free from additional assumptions and therefore is usable with any value of $\Delta Q / Q_{s 0}$. We will use the notation

$$
\mathcal{P}=\frac{\hat{\nu}(\hat{\nu}-\Delta Q)}{Q_{s 0}^{2}}, \quad \mathcal{Q}=\frac{q_{0} \hat{\nu}}{Q_{s 0}^{2}}
$$

to rewrite Eq. (15) in the form

$$
\bar{Y}^{\prime \prime}(\vartheta)+\mathcal{P} \bar{Y}(\vartheta)=\frac{2 \mathcal{Q}}{\pi} \int_{\theta}^{\pi} \bar{Y}\left(\vartheta^{\prime}\right) d \vartheta^{\prime}
$$

which can be reduced to the proper differential equation

$$
\bar{Y}^{\prime \prime \prime}+\mathcal{P} \bar{Y}^{\prime}+\frac{2 \mathcal{Q}}{\pi} \bar{Y}=0 .
$$

A similar equation has been investigated in Ref. [5]. It follows from the paper that, at any real $\mathcal{Q}$, there is an infinite discrete set of the eigenfunctions $\bar{Y}^{(k)}$ with real eigennumbers $\mathcal{P}^{(k)}$ which satisfy the equation. Actually the equation is being solved in this paper step by step with arbitrary value of $\mathcal{Q}$ and some trial value of $\mathcal{P}$, using the initial conditions

$$
\bar{Y}(\pi)=1, \quad \bar{Y}^{\prime}(\pi)=0, \quad \bar{Y}^{\prime \prime}(\pi)=-\mathcal{P}
$$

and coming back to the point $\vartheta=0$. The values of $\mathcal{P}$ assuring the condition $\bar{Y}^{\prime}(0)=0$ have to be separated as the valid eigenvalues. Some of them are plotted in Fig. 4.

The obtained function $\mathcal{P}(Q)$ has to be imaged into the plane $\left(\hat{\nu}, q_{0}\right)$ applying the transformations

$$
\hat{\nu}=\frac{\Delta Q}{2} \pm \sqrt{\frac{\Delta Q^{2}}{4}+\mathcal{P} Q_{0}^{2}}, \quad q_{0}=\frac{Q_{0}^{2} \mathcal{Q}}{\hat{\nu}}
$$

which follow from Eq. (23). Any point of the family represents a real eigentune of the bunch at given SC tune shift. They form several lines representing tunes of different head-tail modes some of which are being shown in Fig. 5. These modes are stable at rather small value of $q_{0}$ because the chromaticity is not included in the consideration. The instability can arise due to coalescence of some neighboring lines at rather large wake field. According to Eq. (22), the condition $d q_{0}=0$ marks the border point where the TMCI threshold appears.

All the tune lines have a well-known form at $\Delta Q=0$ (see, e.g., [7]). Without wake, the eigentunes form set of the multipoles $\nu_{m}=m Q_{s 0}$. Some of them can coalesce at higher $q_{0}$ marking a beginning of the instability region.

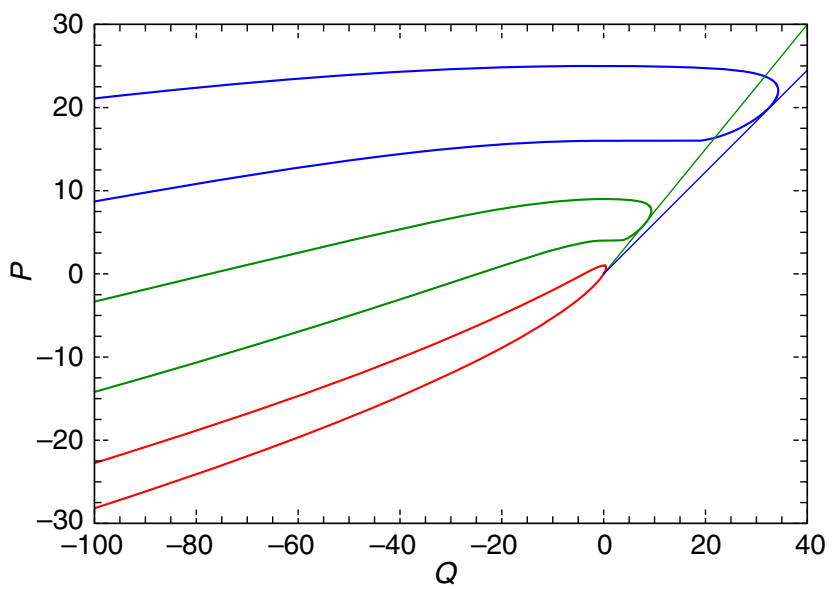

FIG. 4. Lowest real eigennumbers of Eq. (24) and (25). Thin straight lines are tangent to the curves of corresponding color.

Corresponding threshold values of several low TMCI modes are at $\Delta Q=0$

$$
\left(q_{0} / \Omega_{0}\right)_{\text {thresh }}= \pm 0.567, \quad \pm 3.46, \quad \pm 7.37
$$

which agree with Ref. [5]. Other graphs of Fig. 5 illustrate deformation of the tune lines, and movement of the threshold points because of the space charge impact. The picture is very simple with positive wake when the thresholds of all unstable modes monotonously decrease tending to 0 at $\Delta Q \rightarrow \infty$ [5]. Therefore only the cases $q_{0}<0$ are plotted in Fig. 5 at $\Delta Q / Q_{s 0} \geq 6$ and are commented below.

It is seen that the threshold of any unstable mode increases in modulus at increasing $\Delta Q$. However, different modes have different velocity of the movement. The mode caused by coalescence of the multipoles $m=0$ and $m=-1$ is the most unstable at $\Delta Q / Q_{0}<6$. However, threshold of this mode (further marked as $M_{0,-1}$ ) rather rapidly raises with $\Delta Q$ moving to the left and yielding the role of the most unstable mode to $M_{-2,-3}$ at $\Delta Q / Q_{0}=6$. Next mode $M_{-4,-5}$ is more stable at any space charge.

Obtained thresholds of these coupled modes are plotted against the SC tune shift in Fig. 6. Results of the expansion technique with $N_{\max }=19$ are added to the plot being shown by the short black line. There is a perfect coincidence of the results at $\Delta Q / Q_{0} \leq 15$ that is in the applicability region of the expansion technique, as it has been specified above.

It follows from Fig. 5 that the tunes of the potentially unstable modes satisfy the condition $|\hat{\nu}| \ll \Delta Q$ at $\Delta Q / Q_{s 0} \gg 1$. One can see that similar result occur only under the condition $|\mathcal{P}| \ll \Delta Q / Q_{s 0}$ when Eq. (27) leads to the expressions

$$
\hat{\nu} \simeq-\frac{\mathcal{P} Q_{s 0}^{2}}{\Delta Q}, \quad q_{0} \simeq-\frac{\mathcal{Q}}{\mathcal{P}} \Delta Q
$$



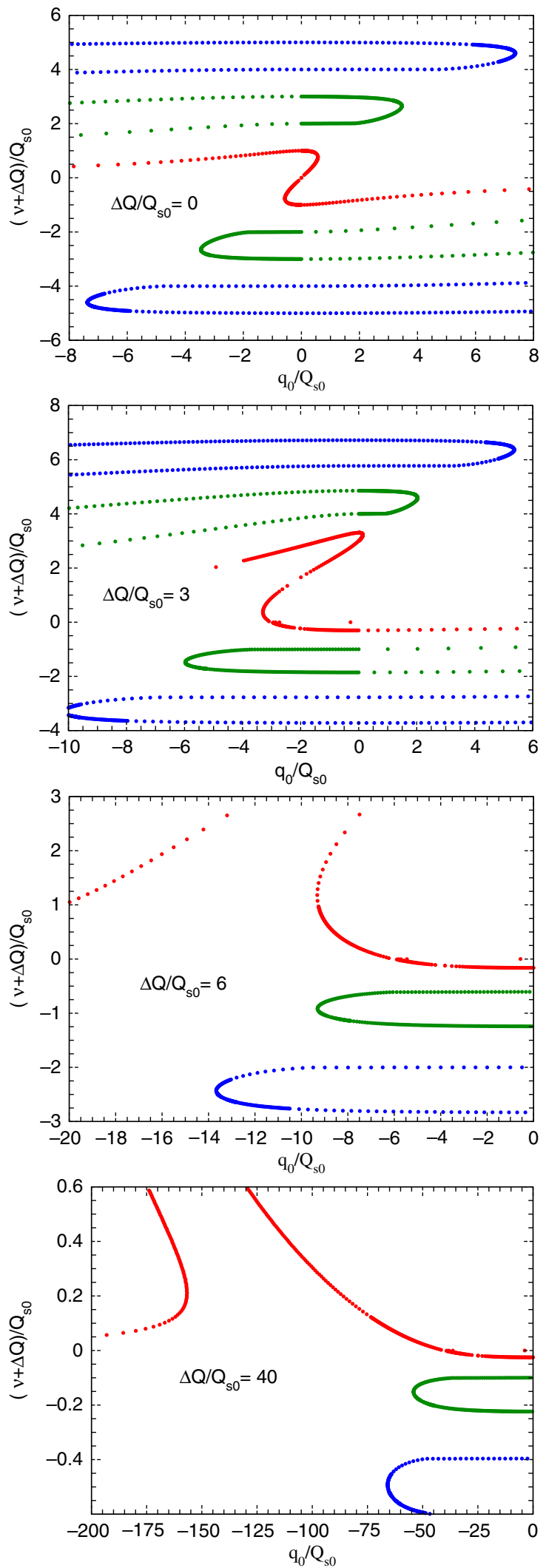

FIG. 5. The bunch head-tail modes against the wake strength at different SC tune shifts. The extremal points where $d q_{0}=0$ mark the beginning of the instability region. Only negative wakes are displayed at $\Delta Q / Q_{s 0} \geq 6$.

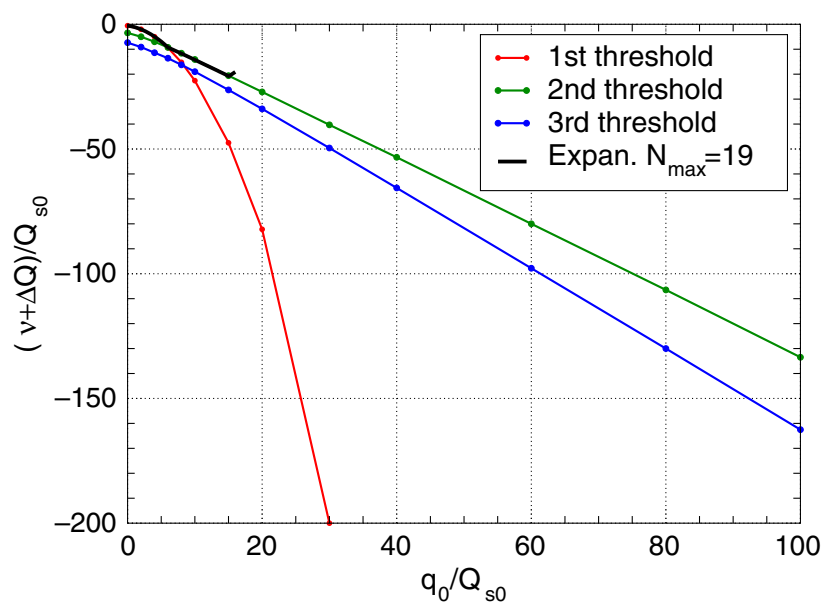

FIG. 6. Instability threshold of the lowest TMCI modes. The short black line is copied from Fig. 1 representing result of the expansion technique at $N_{\max }=19$. Its deviation from the current lowest mode does not exceed $1 \%$ at $\Delta Q / Q_{s 0}<15$.

According to these equations, the value of $q_{0}$ varies when the point $(\mathcal{Q}, P)$ moves along one of the curves in Fig. 4. The TMCI threshold obtained by Eq. (22) appears in the point where the condition $d q_{0}=0$ is fulfilled, that is $d \mathcal{P} / P=d \mathcal{Q} / Q$. It is the point of tangency of the curve with the straight line $\mathcal{P}=k \mathcal{Q}$ where $k$ is a constant. These tangents are shown in Fig. 4 by green and blue straight lines providing $k_{g}=0.75$ and $k_{b}=0.61$. Therefore the asymptotic TMCI thresholds of corresponding modes are

$$
\left(q_{0}\right)_{g}=-1.33 \Delta Q, \quad\left(q_{0}\right)_{b}=-1.63 \Delta Q,
$$

in agreement with Fig. 6. Note that the procedure is unfit for the red line resulting in $k_{r}=0 / 0$, because $\hat{\nu} \nrightarrow 0$ in the case.

\section{RESISTIVE WALL WAKE}

The resistive wall is the most common and important source of transverse instability in circular accelerators. Its wake function reaches a maximum at the distance $z=b / \gamma$ from the source with $b$ as the beam pipe radius. If the bunch length satisfies the condition $z_{b} \gg b / \gamma$, and the wall is thick, the simplest relation for the transverse wake function is applicable:

$$
W_{1}(z)=-\frac{4 R}{b^{3}} \sqrt{\frac{c}{\sigma|z|}}
$$

where $\sigma$ is the pipe wall conductivity (see, e.g., [7]). According to Eqs. (4) and (5), the corresponding basic tune shift is:

$$
\nu_{\text {rigid }}=q_{0}=-\frac{4 r_{0} R^{2} N_{b}}{3 \pi \beta \gamma b^{3} Q_{c}} \sqrt{\frac{c}{\sigma z_{b}}} .
$$


Therefore, in agreement with the accepted conditions, the normalized wake function in Eq. (11) is

$$
q\left(\vartheta^{\prime}-\vartheta\right)=\frac{q_{0} \kappa}{\sqrt{\vartheta^{\prime}-\vartheta}}, \quad \kappa=\frac{3 \sqrt{\pi}}{8}
$$

Instability of similar bunch was considered in Ref. [6] using the expansion technique at $\Delta Q / Q_{s 0} \leq 9$. Now we will investigate the problem without this restriction using equation like Eq. (24) which provides in the case

$$
\begin{aligned}
\bar{Y}^{\prime \prime}(\vartheta)+\mathcal{P} \bar{Y}(\vartheta) & =\frac{2 \mathcal{Q} \kappa}{\pi} \int_{\vartheta}^{\pi} \frac{\bar{Y}\left(\vartheta^{\prime}\right) d \vartheta^{\prime}}{\sqrt{\vartheta^{\prime}-\vartheta}}, \\
\bar{Y}^{\prime}(0) & =\bar{Y}^{\prime}(\pi)=0 .
\end{aligned}
$$

In contrast with Eq. (24), this equation is not reducible to the pure differential form like Eq. (25). Nevertheless, the step-by-step method of the solution is applicable as above being enhanced by calculation of the integral. The result is represented in Fig. 7 where six lower eigennumbers of the equation are shown.

These curves are so similar to those in Fig. 4 that there is no need to plot the tune lines like Fig. 5. The statement pertains equally to the possibility to determine the asymptotic behavior of the threshold by the build-up of a tangent to the green line. Therefore we represent in Fig. 8 only the net result, namely the TMCI threshold with the resistive wall wake against the SC tune shift. At $\Delta Q=0$, the threshold is a little larger in comparison with the square wake: $\left(q_{0}\right)_{\text {thresh }} / Q_{s 0} \simeq-0.90$ instead of -0.57 . However, henceforth it grows slower having asymptotically $\left(q_{0}\right)_{\text {thresh }} \simeq-1.1 \Delta Q$ instead of $-1.33 \Delta Q$.

It is necessary to take into account that the resistive wake falls rather slowly so it can reach the neighboring bunch or turn and provoke a multibunch/multiturn instability. The problem was considered in Ref. [5] leading to the conclusion that the TMCI effect prevails at the condition

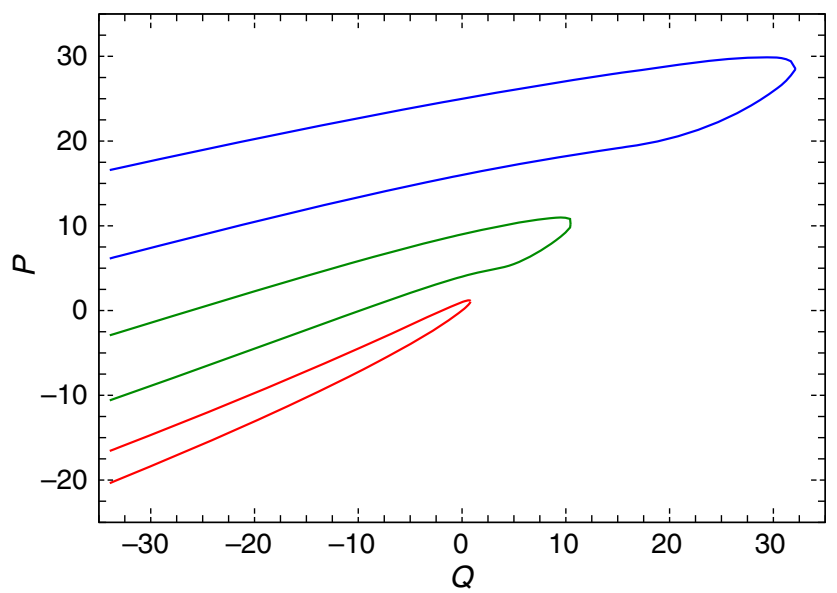

FIG. 7. Lowest real eigennumbers of the resistive wall wake, Eq. (33).

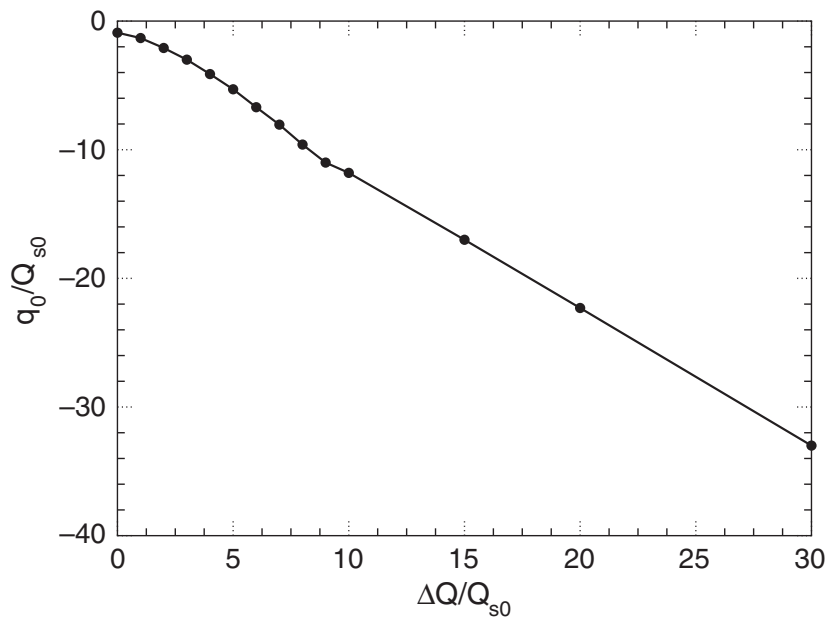

FIG. 8. TMCI threshold of the resistive wall wake. Its asymptotic value is: $q_{0}=-1.1 \Delta Q$.

$$
2\left(h-\frac{0.35}{\sqrt{h}}\right) \sqrt{\frac{\sigma_{z}}{2 \pi R}}<1
$$

where $h$ is number of bunches, and $\sigma_{z}$ is their rms length.

\section{EXPONENTIAL WAKE}

The exponential wake of the form

$$
\begin{aligned}
q(\vartheta) & =\kappa q_{0} \exp (-\alpha \vartheta), \\
\kappa & =\frac{\pi \alpha}{2}\left[1-\frac{1-\exp (-\pi \alpha)}{\pi \alpha}\right]^{-1}
\end{aligned}
$$

is considered in this section. Coefficient $\kappa$ is added to meet the requirement $\nu_{\text {rigid }}=q_{0}$ with any $\alpha$. It is assumed as well that the wake decays rather fast after the bunch end, so that it cannot reach the following bunch or turn.

Substitution of this expression into Eq. (11) results in the equation

$$
\bar{Y}^{\prime \prime}(\theta)+\mathcal{P} \bar{Y}(\theta)=\frac{2 \kappa \mathcal{Q}}{\pi} \exp (\alpha \theta) \int_{\theta}^{\pi} \bar{Y}\left(\theta^{\prime}\right) \exp \left(-\alpha \theta^{\prime}\right) d \theta^{\prime}
$$

where the notations embedded by Eq. (23) are used. It can be reduced to the proper differential form like Eq. (25) with the boundary conditions like Eq. (26)

$$
\begin{array}{r}
\bar{Y}^{\prime \prime \prime}-\alpha \bar{Y}^{\prime \prime}+\mathcal{P} \bar{Y}^{\prime}+\left(\frac{2 \kappa \mathcal{Q}}{\pi}-\alpha \mathcal{P}\right) \bar{Y}=0, \\
\bar{Y}(\pi)=1, \quad \bar{Y}^{\prime}(\pi)=0, \quad \bar{Y}^{\prime \prime}(\pi)=-\mathcal{P}, \quad \bar{Y}^{\prime}(0)=0 .
\end{array}
$$

Several solutions of this equation are shown in Fig. 9 and Fig. 10 at $\pi \alpha=0,5,10,15,20$. The lowest eigennumbers 


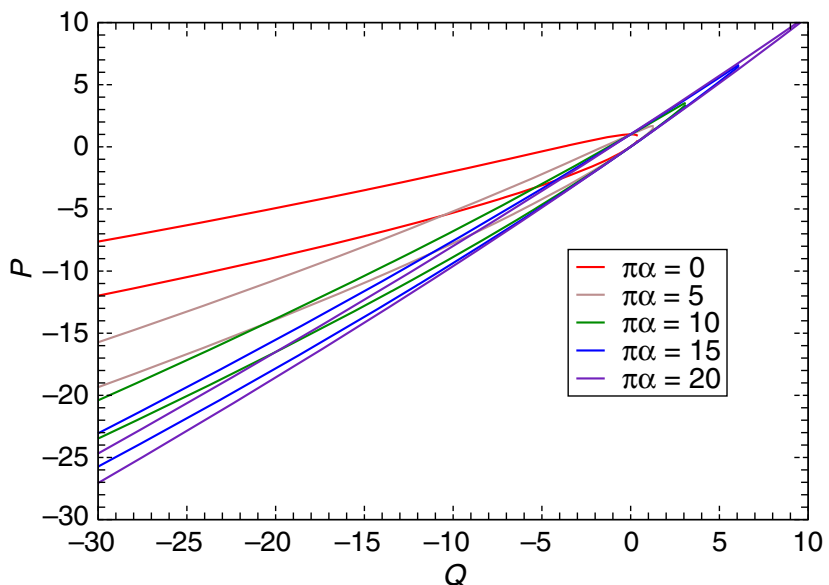

FIG. 9. The lowest real eigennumbers of Eq. (30) at different $\alpha$.

which are represented in Fig. 9 are the analogues of the red lines in Fig. 4. The higher eigennumbers are plotted in Fig. 10 in the restricted region $\mathcal{Q}>0$, where the instability of higher modes can have a start, as it has been shown in Sec. III B.

The bunch eigentunes can be obtained at any SC tune shift with help of Eq. (27) applied to corresponding curve of Fig. 9 or Fig. 10. The lowest eigentunes are represented in Figs. 11 and 12. The first of them demonstrates strong increase of the threshold when the damping coefficient increases at $\Delta Q=0$. However, the dependence becomes weaker at higher SC tune shift as illustrated by Fig. 12 at $\Delta Q / Q_{s 0}=4$. There are additional data in Table II where the threshold of this mode is represented in the interval $\Delta Q / Q_{s 0} \leq 6$. It is seen that, at $\Delta Q / Q_{s 0}>\sim 5$, the threshold goes down at higher $\alpha$.

The behavior of the higher modes does not greatly depend on $\alpha$, and is rather well illustrated by green and blue lines in Fig. 5. The mode produced by the coalescence of the multipoles $m=-1$ and -2 becomes the most unstable at $\Delta Q / Q_{s 0}>5-10$, depending on $\alpha$. The general picture is shown in Fig. 13, and it demonstrates that the

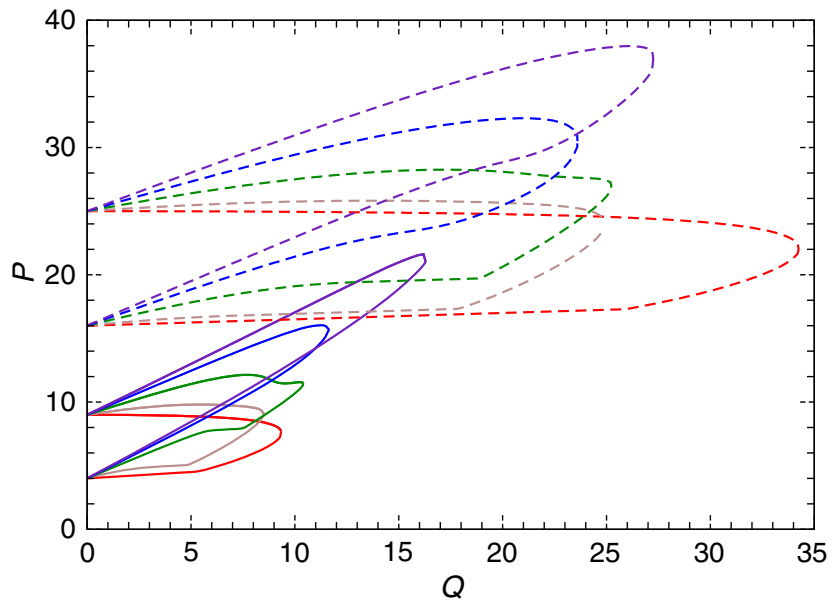

FIG. 10. Higher real eigennumbers of Eq. (34) at different $\alpha$.

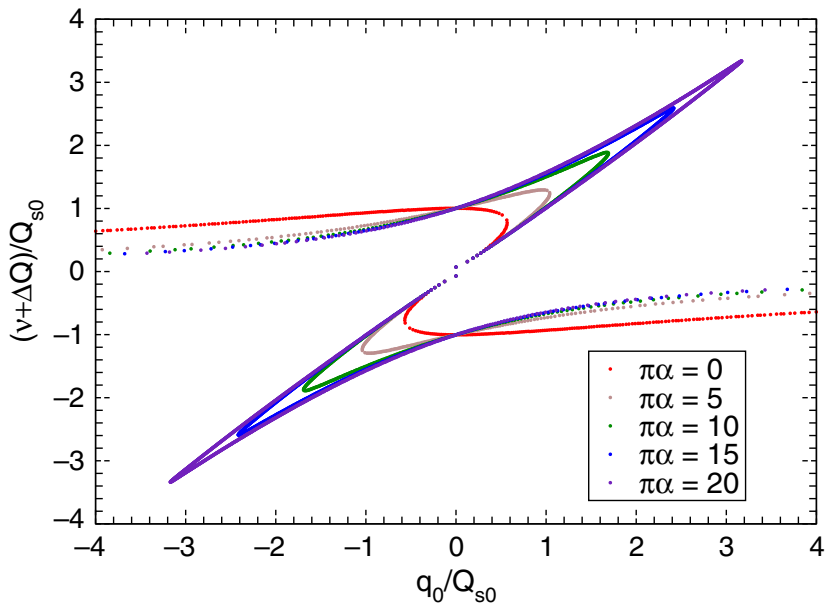

FIG. 11. Lowest eigentunes of the bunch against the exponential wake strength at $\Delta Q=0$.

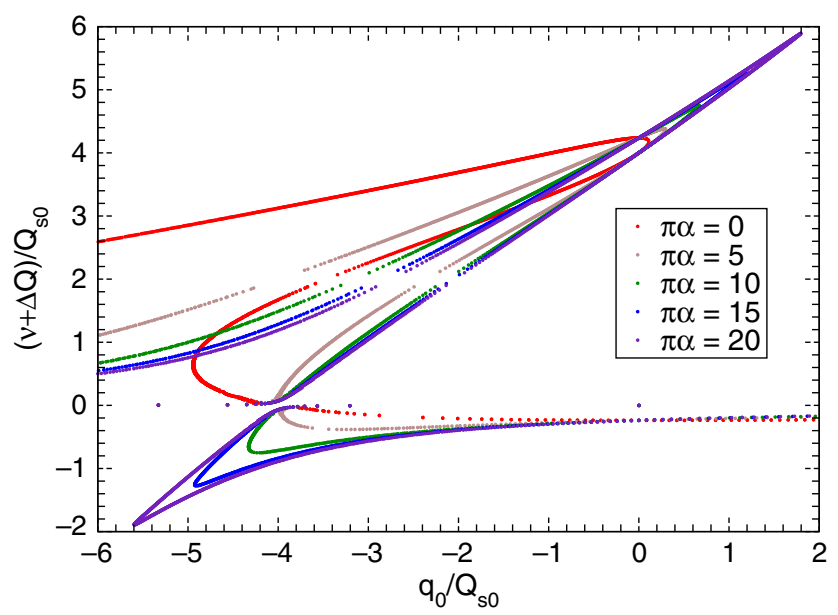

FIG. 12. Lowest eigentunes of the bunch against the exponential wake strength at $\Delta Q=4$.

dependence of the threshold on the wake strength is almost linear at $\Delta Q / Q_{s 0}>10$.

The asymptotic behavior of the threshold can be obtained by plotting of the tangent to the curves in Fig. 10, as it has been explained at the end of Sec. III. The asymptotic formula is

$$
\left(q_{0}\right)_{\text {tresh }}=k(\alpha) \Delta Q
$$

TABLE II. The TMCI threshold of the exponential wake due to a coalescence of the lowest multipoles $m=0$ and $m=-1$ at modest space charge tine shift.

\begin{tabular}{lccccccc}
\hline \hline$\Delta Q / Q_{s 0} \rightarrow$ & 0 & 1 & 2 & 3 & 4 & 5 & 6 \\
\hline$\pi \alpha=0$ & -0.57 & -1.10 & -2.00 & -3.30 & -4.95 & -6.95 & -9.29 \\
$\pi \alpha=5$ & -1.03 & -1.47 & -2.23 & -3.04 & -4.00 & -5.08 & -6.20 \\
$\pi \alpha=10$ & -1.71 & -2.19 & -2.80 & -3.55 & -4.34 & -5.18 & -6.07 \\
$\pi \alpha=15$ & -2.42 & -2.91 & -3.52 & -4.18 & -4.82 & -5.40 & -6.03 \\
$\pi \alpha=20$ & -3.20 & -3.69 & -4.28 & -4.90 & -5.45 & -6.00 & -6.60 \\
\hline \hline
\end{tabular}




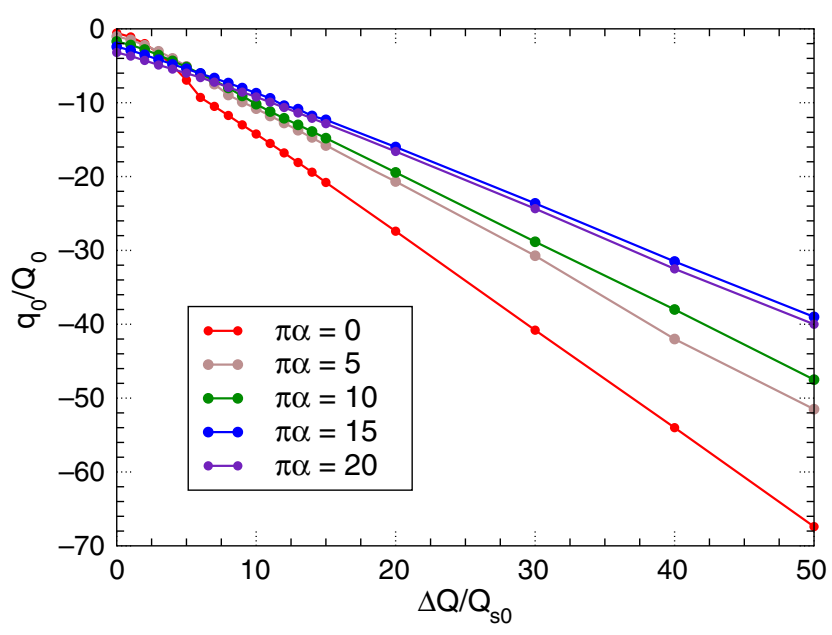

FIG. 13. Instability threshold of exponential wake with different $\alpha$ against the SC tune shift.

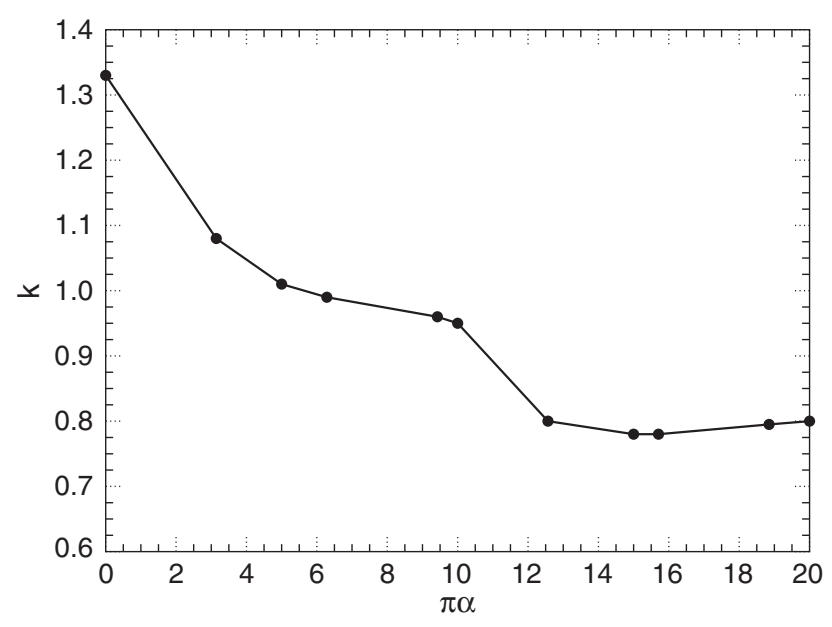

FIG. 14. Asymptotic ratio $q_{0} / \Delta Q$ of exponential wake against the damp coefficient.

with coefficients $k(\alpha)$ given by Fig 14 . According to this plot, dependence of the TMCI threshold on the damping factor is not very strong and is almost constant at $\pi \alpha \geq 12$. It happens due to normalization which has been used in Eq. (34) to reach $\nu_{\text {rigid }}=q_{0}$. The much stronger dependence has been obtained in Ref. [1] because the relation $\kappa=2$ has been actually used there. With this correction, the results are rather close at $\Delta Q / Q_{s 0}<20$.

\section{CONCLUSIONS}

The transverse mode coupling instability is considered in the paper in framework of the hollow bunch model in a square potential well with space charge tune shift taken into account. Two methods are used to calculate the instability threshold.

The first of them is built upon the expansion technique using an infinite set of basis functions with subsequent truncations of the series. The similar approximation method was used by different authors before to analyze the bunch instability in a parabolic potential well, whose circumstance allows to compare the results. The obtained qualitative and quantitative coincidence allows to conclude that region of applicability of the square well model for the TMCI description is wider than it is originally assumed, because it correctly describes the lowest radial modes which coalescence is just responsible for the instability.

However, the expansion method is actually applicable at a moderate value of the space charge tune shift. Therefore, another method is also proposed and applied in the paper consisting in a direct step-by-step solution of integraldifferential equation for the bunch offset with space charge. It confirms correct results of the expansion method in the area of its applicability, and it allows them to arbitrary large space charge. The method is applied with the square, resistive, and various exponential wake forms. In all the cases, the rather similar results are obtained for the normalized wake amplitudes $q_{0}$ if the tune shift of the lowest (rigid) head-tail mode is used each time as the scaling factor. In particular, it is shown that the instability threshold is asymptotically proportional to the tune shift: $\left(q_{0}\right)_{\text {thresh }}=k \Delta Q$ with the coefficient $k=0.8-1.3$, dependent on the wake form.

The results allow us to conclude that properly normalized square wake can be a quite appropriate model for monotonous wake functions. As the SC tune shift increases, the TMCI threshold of the negative wake tends to $\infty$ but the threshold of the positive wake tends to 0 . However, the problem remains open in the case of oscillating wake including its asymptotic behavior.

\section{ACKNOWLEDGMENT}

Fermi National Accelerator Laboratory is operated by Fermi Research Alliance, LLC under Contract No. DEAC0207CH11395 with the U.S. Department of Energy.

[1] M. Blaskiewicz, Fast head-tail instability with space charge, Phys. Rev. ST Accel. Beams 1, 044201 (1998).

[2] A. Burov, Head-tail modes for strong space charge, Phys. Rev. ST Accel. Beams 12, 044202 (2009); Phys. Rev. ST Accel. Beams 12, 109901 (2009).

[3] V. Balbekov, Transverse instability of a bunched beam with space charge and wakefield, Phys. Rev. ST Accel. Beams 14, 094401 (2011).

[4] M. Blaskiewicz, in Proceedings of the 3rd International Particle Accelerator Conference, New Orleans, LA, 2012 (IEEE, Piscataway, NJ, 2012).

[5] V. Balbekov, Single bunch transverse instability in a circular accelerator with chromaticty and space charge, J. Instrum. 10, P10032 (2015).

[6] V. Balbekov, Transverse mode coupling instability with space charge and different wakefields, Phys. Rev. Accel. Beams 20, 034401 (2017).

[7] B. Ng, Report No. Fermilab-FN-07-13, 2002. 